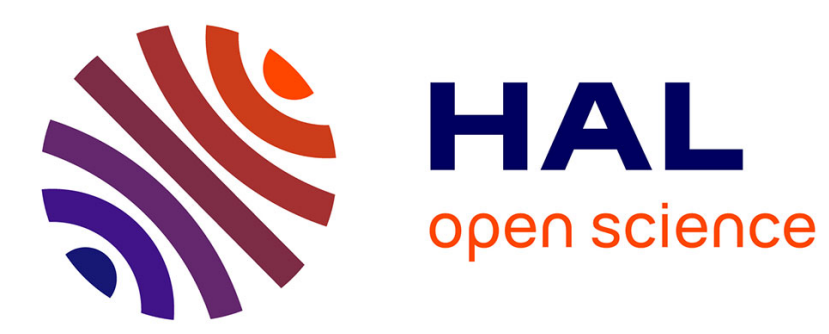

\title{
L'impact de la position phrastique sur les fonctions et valeurs des SP adverbiaux: l'exemple des SP en sur et dans
}

\author{
Laure Sarda, Shirley Carter-Thomas
}

\section{- To cite this version:}

Laure Sarda, Shirley Carter-Thomas. L'impact de la position phrastique sur les fonctions et valeurs des SP adverbiaux: l'exemple des SP en sur et dans. Travaux de Linguistique: Revue Internationale de Linguistique Française, 2012, 64, pp.21-54. halshs-00664075

\section{HAL Id: halshs-00664075 \\ https://shs.hal.science/halshs-00664075}

Submitted on 28 Jan 2012

HAL is a multi-disciplinary open access archive for the deposit and dissemination of scientific research documents, whether they are published or not. The documents may come from teaching and research institutions in France or abroad, or from public or private research centers.
L'archive ouverte pluridisciplinaire HAL, est destinée au dépôt et à la diffusion de documents scientifiques de niveau recherche, publiés ou non, émanant des établissements d'enseignement et de recherche français ou étrangers, des laboratoires publics ou privés. 


\section{Pre-publication draft}

\section{Laure Sarda}

Shirley Carter-Thomas

\section{L'impact de la position phrastique sur les fonctions et valeurs des SP adverbiaux : l'exemple des SP en Sur et Dans}

Abstract

The objective of this article is to examine the impact of sentence position on the values and functions of prepositional phrases (PPs) with sur (on) and dans (in). The first part focuses on the range of meanings these PPs carry. We consider to what extent specific semantic values are correlated with sentence position. This is followed by an analysis of the different syntactic roles fulfilled by the PPs. We consider to what extent their semantic values are associated with particular syntactic functions and propose a classification of PPs which includes semantic, syntactic and pragmatic criteria. Situating our study within Charolle's theory of 'Discourse frames' (cf. among others Charolles 1997, Charolles \& Pery-Woodley 2005), we examine in a third part the potential textual and organising functions of PPs and contrast this type of structuring with a rather different type of structuring based on thematic progression.

\section{Résumé}

L'objectif de cet article est d'étudier l'impact de la position phrastique (i.e. initiale vs. finale) sur les valeurs et les fonctions que peuvent prendre les SP en dans et sur. Dans la première partie nous nous focaliserons sur la valeur sémantique de ces SP. Nous observerons dans quelle mesure certaines valeurs sémantiques peuvent apparaitre plus régulièrement dans certaines positions. La $2^{\text {ème }}$ partie propose une analyse des différentes fonctions syntaxiques que peuvent prendre les SP. Nous observons les corrélations entre valeur sémantique et statut syntaxique et proposons une classification des SP basée sur des critères sémantiques, syntaxiques et pragmatiques. Dans la lignée des travaux sur l'encadrement du discours (cf. entre autres Charolles 1997, Charolles \& Pery-Woddley 2005), nous examinons dans la troisième partie les fonctions textuelles et organisatrices des SP. L'accent est mis sur la différence entre la structuration cadrative et la structuration thématique. 
Le français moderne fait partie des langues SVO où l'ordre des mots est relativement fixe. Les adverbiaux jouissent toutefois d'une mobilité particulière. Ils peuvent en effet être antéposés, insérés ou bien postposés. Ce positionnement n'est pas anodin mais répond à des contraintes syntaxiques et/ou textuelles qui peuvent avoir à leur tour une influence importante sur l'interprétation de la phrase. L'éventail des valeurs sémantiques assumées par les différents SP (syntagmes prépositionnels) adverbiaux que nous considérons ici est également très vaste. Les SP adverbiaux en sur et dans peuvent assumer, en plus de leurs valeurs usuelles, spatiales et temporelles, toute une gamme de valeurs que nous avons qualifiées de notionnelles :

[1] Dans la plupart des cas, j'ignorais le montant du marché. ;

[2] Comme la torture n'était ni reconnue, ni officialisée, la comparution devant un tribunal de guerre servait surtout d'intimidation, mais elle était exceptionnelle dans la réalité.

[3] Sur ce dernier point, rien à dire : (...)

[4] Le groupe annonce la sortie de 67 modèles d'ici à 2005 sur l'ensemble de son portefeuille de marques.

L'objectif de cet article est d'étudier les corrélations entre (i) la position phrastique (initiale vs. finale), (ii) les valeurs sémantiques et (iii) les fonctions textuelles que peuvent prendre les SP en dans et sur. L'étude est basée sur le corpus journalistique (Le Monde, décembre 2000) exploité pour construire la base $\mathrm{BSP}^{1}$ à partir de laquelle nous avons constitué un sous-corpus d'analyse de 660 exemples de sur et dans en position initiale (tête) et finale (queue).

Les théories sur le statut spécial de la position initiale sont nombreuses. Pour Givon (1988), la position initiale correspond à la position la plus saillante sur le plan cognitif, point de vue confirmé par des études psycholinguistiques (VanPatten 2004); pour Halliday (1994), la position initiale est associée au thème phrastique. Virtanen (2004 : 80) la caractérise par trois fonctions importantes: celle de point d'ancrage, de point d'orientation pour le segment à venir ou d'indicateur d'informations cruciales. Il convient aussi de rappeler le fait que le placement

\footnotetext{
${ }^{1}$ La base BSP (Base des Syntagmes Prépositionnels) a été constituée dans le cadre du projet SFA (Spatial Framing Adverbial), soutenu par l'Agence Nationale de la Recherche. Elle contient plus de 18000 SP annotés syntaxiquement (à l'aide de SYNTEX), et sémantiquement par les collaborateurs du projet (cf. Présentation du volume).
} 
à l'initiale de la phrase libère la place à la fin de phrase, pour les éléments rhématiques qui permettent au texte de progresser (Firbas 1992, Mathesius 1975).

Comme le constate Lambrecht, le fait que les linguistes confèrent à la position initiale plusieurs fonctions potentielles n'est pas contradictoire :

Given that sentence initial position is cognitively speaking an eminently salient position, it would be surprising if the prominence associated with this position could be exploited for a single function (...). (1994: 201)

Il serait donc raisonnable de postuler que l'agencement de la phrase correspond aux besoins communicatifs du lecteur dans une situation donnée. Virtanen (1992) identifie par exemple un certain nombre de stratégies textuelles permettant à un locuteur d'organiser son texte. Si l'auteur choisit d'organiser ses propos autour d'une chronologie temporelle, on doit s'attendre à trouver un adverbial temporel en première position. Toutefois, la liberté de placement des constituants n'est pas totale, car les contraintes pesant sur l'ordre des mots sont diverses. Enkvist $(1976$; $1985 ; 1991)$ identifie en plus de la syntaxe et de la structure sémantique, des contraintes liées à l'iconicité expérientielle, à la lourdeur des constituants et au traitement de l'information en temps réel. De plus, le poids accordé à ces différents facteurs et leurs interactions vont varier selon les situations de communication (oral/écrit) et selon les structures des langues concernées.

Dans cet article, nous accordons une attention particulière aux fonctions organisatrices des SP étudiés, à leur impact au niveau de la structure du discours. Notre réflexion s'insère dans la lignée des travaux sur les adverbiaux dits cadratifs (cf. Charolles 1997, Charolles \& Prévost 2003, Charolles \& Pery-Woddley 2005). Ces travaux ont mis en évidence le fait que les adverbiaux antéposés sont potentiellement dotés d'un portée extraphrastique. Autrement dit, lorsqu'ils sont en tête de phrase, certains adverbiaux peuvent prendre des fonctions organisatrices qu'ils n'auraient pas en queue de phrase. Dans cette perspective, nous proposons de comparer les portées respectives des SP initiaux et finaux en sur et dans.

Il est légitime de réexaminer cette question, en se fondant sur un corpus, dans la mesure où Crompton (2006) minimise le rôle structurant des adverbiaux préposés, à l'encontre d'un grand nombre d'études sur la question (cf. entre autres : Charolles 1997 ; Diessel 2001, 2005, 2008 ; Goutsos 1996 ; Haider 2004 ; Ho-Dac \& Péry-Woodley 2009 ; Lowe 1987 ; Ramsay 1987 ; Thomson 1985 ; Thompson \& Longacre 1985 ; Verstraete 2004, Virtanen 1992). Crompton défend l'idée selon laquelle les adverbiaux en position finale jouissent du même pouvoir que les préposés : la position phrastique n'est pas selon lui le facteur décisif ; il soutient en particulier que les adverbiaux en position finale ont la même capacité que les 4 
initiaux à étendre leur portée au-delà de leur phrase d'accueil, et à participer ainsi à la structuration du discours. Or notre hypothèse est que la position phrastique exerce une influence forte non seulement sur les valeurs sémantiques véhiculées par ces adverbiaux mais aussi sur leurs capacités à jouer un rôle organisationnel au niveau textuel. Nous nous attacherons à mettre en évidence dans ce travail deux phénomènes distincts : une structuration cadrative strictement liée à la position initiale vs. un type de progression rhématique déclenchant une relation d'élaboration. Dans le premier cas, les SP définissent le fond (ground) ou arrière-plan qui établit un critère sémantique pour l'interprétation des phrases à venir ; dans le second, le SP en position rhématique s'apparente à un hyper-rhème qui peut déclencher une relation d'élaboration (i.e. une description des sous-événements qui le composent).

L'organisation de l'article est la suivante. Dans une première partie, nous observons les corrélations entre sens et position des SP. Dans une deuxième partie, nous essayons de voir dans quelle mesure certaines valeurs sémantiques peuvent être associées plus régulièrement à certaines fonctions syntaxiques. Nous proposons une classification des SP à l'interface entre syntaxe, sémantique et pragmatique. Enfin, nous portons notre attention sur les fonctions textuelles ou organisatrices de ces SP lorsqu'ils ont une fonction adverbiale. Ces fonctions organisatrices dépendent-t-elles (i) de la valeur propre de chaque préposition, (ii) de la valeur sémantique du groupe prépositionnel (iii) ou de la position initiale elle-même qui encode certaines instructions pour l'interprétation du discours?

\section{Corrélations entre sens et positions}

Cette partie est centrée sur une présentation quantitative des données observées dans notre corpus. Nous examinons dans quelle mesure certaines valeurs sémantiques peuvent apparaitre plus régulièrement dans certaines positions. Nous commençons par considérer la répartition sémantique des SP (quel sens dans quelle position), puis nous donnons une description plus détaillée des différents usages annotés comme 'notionnels'.

\subsection{Répartition sémantique brute}

L'annotation sémantique a consisté à attribuer à chaque occurrence de SP une primitive (parmi 5 possibles : espace, temps, notionnel, cause, énonciatif) et une valeur sémantique (parmi 14 possibles: source, localisation, but, durée, moyen, manière, qualification, 
quantification, à propos, support, modalisation du dire, forme revêtue, cause, temporel). La dissociation des primitives et des valeurs sémantiques qui leur sont associées permet d'effectuer des tris à deux échelles de spécification différentes.

Nous pouvons ainsi faire apparaître de la proportion des emplois des SP en sur et dans pour chaque primitive. Les résultats sont calculés en pourcentage sur le total d'occurrences de chaque préposition. Les deux prépositions ont un profil globalement assez semblable. Dans présente $67 \%$ d'usages notionnels, $27 \%$ spatiaux et seulement $4 \%$ temporels. Sur présente $70 \%$ d'usages notionnels, $22 \%$ spatiaux et $6 \%$ temporels.

Le tableau 1 détaille la fréquence d'apparition en tête vs en queue pour chaque primitive sémantique. Elle est calculée ici en pourcentage sur le total d'occurrence de chaque préposition.

\begin{tabular}{ccccccc}
\hline & \multicolumn{7}{c}{ Primitives } & \\
\hline Préposition & Position & Espace & Temps & Notionnel & Enonciatif & Cause \\
\hline Dans & Tête & 8 & 1 & 20 & 0 & 0 \\
& Queue & 19 & 3 & 47 & 0 & 1 \\
& Total & $\mathbf{2 7}$ & $\mathbf{4}$ & $\mathbf{6 7}$ & $\mathbf{0}$ & $\mathbf{1}$ \\
\hline \multirow{2}{*}{ Sur } & Tête & 11 & 2 & 21 & 1 & 1 \\
& Queue & 11 & 4 & 49 & 0 & 1 \\
& Total & $\mathbf{2 2}$ & $\mathbf{6}$ & $\mathbf{7 0}$ & $\mathbf{1}$ & $\mathbf{2}$ \\
\hline
\end{tabular}

Tableau 1 : Distribution des SP en tête vs. queue par primitive sémantique en \% sur le total d'occurrences de chaque préposition

A partir de ces résultats, on a pu établir, dans le tableau 2 ci-dessous, que la répartition des usages des SP en dans est de $70 \%$ en queue et $30 \%$ en tête, tant pour les usages spatiaux que notionnels. En ce qui concerne les SP en sur, on observe d'une part une répartition homogène (50/50) entre les positions tête et queue pour les usages spatiaux. On remarque d'autre part que de façon équivalente à la distribution des usages de dans, les usages notionnels de sur apparaissent dans $70 \%$ des cas en queue et $30 \%$ en tête. Par ailleurs, le faible nombre d'occurrences pour les valeurs temporelle, énonciative et cause avec dans et sur, ne permet pas de tirer des conclusions statistiques.

\begin{tabular}{ccccc}
\hline & \multicolumn{2}{c}{ Espace } & \multicolumn{2}{c}{ Notionnel } \\
\hline En \% & Dans & Sur & dans & sur \\
Tête & 30 & 50 & 30 & 30 \\
Queue & 70 & 50 & 70 & 70 \\
\hline
\end{tabular}

Tableau 2 : Distribution des SP en tête vs. queue sur le total d'occurrences par primitive sémantique 
Il est donc frappant de constater que dans les deux catégories sémantiques les plus fréquentes (espace et notionnel), la distribution des deux prépositions est la même (30\% en tête/ $70 \%$ en queue) à l'exception des usages spatiaux de sur qui apparaissent autant en tête qu'en queue.

Devant la proportion importante d'usages notionnels (autour de 70\%), nous avons observé de plus près la spécification de ces usages par les valeurs sémantiques.

\subsection{Analyse des emplois notionnels}

Les valeurs sémantiques associées aux emplois notionnels des syntagmes prépositionnels en sur et dans sont listées dans les colonnes du tableau 3 ci-dessous :

\begin{tabular}{llllllll}
\hline & localisation & $\grave{a}$ propos & support & quantification & manière & But & (autre) \\
\hline Dans & 68 & 0 & 11 & 0 & 5 & 7 & 9 \\
Sur & 35 & 40 & 0 & 9 & 8 & 2 & 6 \\
Total & 51 & 21 & 6 & 5 & 7 & 5 & 5 \\
\hline
\end{tabular}

Tableau 3 : Spécification des emplois notionnels des SP en sur et dans (en pourcentage)

Les usages de dans 'notionnel' sont, dans $68 \%$ des cas, spécifiés par la valeur 'localisation'. Cette combinatoire 'notionnel-localisation' est apparentée à ce qui est décrit dans la littérature par la notion d'espace métaphorique, elle est illustrée par les exemples [5-6]. Loin derrière mais en proportion non négligeable (11\%) arrivent les emplois spécifiés par la valeur 'support', qui désigne une forme de localisation de la source ou du support d'un discours (cf. [7]). Ces emplois sont caractérisés par la présence d'un verbe de parole dans la proposition.

[5] Il est donc utilisé, hors de son sens concret, dans un débat intérieur français.

[6] Ce résultat, annoncé mercredi 29 novembre, est surtout un signal important dans le marché de l'équipement des villes.

[7] Dans une "lettre ouverte ", publiée le 25 novembre par le quotidien France Guyane, il explique que le débat auquel il a convié les élus et les partis politiques représentatifs, le 18 décembre, doit «s'ouvrir sans tabou, ni exclusive ».

Pour les emplois notionnels des SP en sur, on trouve 35\% d'usages ayant une valeur de localisation (cf.[8]), 40\% d'usages ayant une valeur 'à propos', spécifique à sur, et illustrée en [9] ; puis 9\% d'une autre valeur spécifique à sur, la valeur 'quantification' illustrée en [10], et enfin $8 \%$ de la valeur 'manière' illustrée en [11]

[8] Sur la bande son, une voix présentée comme celle de M. Koutchma suggère d'écarter M. Gongadze en organisant son enlèvement en Géorgie par des Tchétchènes 
[9] Sur la peine de mort, l'avortement, le non-contrôle des ventes d'armes, George W. Bush a déjà montré au Texas qu'il se situait à l'extrême droite de l'échiquier politique.

[10] Sur les 78 boîtes de nuit testées, l'association a constaté de "flagrants délits de discrimination $»$ raciales dans 31 cas.

[11] Samedi, la manifestation s'est achevée devant l'hôtel de ville, sur une note d'espoir.

Le tableau 4 ci-dessous montre la proportion (en pourcentage) des usages notionnels en tête ou en queue des SP en sur et dans. Seules les valeurs 'localisation', et (dans de bien moindres proportions) 'manière' sont portées par les deux prépositions et dans les deux positions. La préposition sur se démarque par le fait qu'elle apparait avec des valeurs très spécifiques : 'à propos' et 'quantification'; ces deux valeurs présentent des préférences inverses pour les positions tête et queue. Comme on peut l'observer dans le tableau la valeur 'à propos' est dans $30 \%$ des cas en tête et la valeur 'quantification' dans $75 \%$ des cas en tête.

\begin{tabular}{ccccccc}
\hline & & à propos & localisation & Manière & Quantification & Support \\
\cline { 3 - 7 } & & 29 & 17 & & 72 \\
\cline { 3 - 7 } Dans & Tête & 71 & 83 & & \\
\hline \hline \multirow{2}{*}{ Sur } & Queue & 30 & 20 & 37 & 75 & \\
& Queue & 70 & 80 & 63 & 25 & \\
\hline
\end{tabular}

Tableau 4 : Proportion d'occurrences en tête/ queue des valeurs sémantiques associées à la primitive notionnelle en \% (calculé pour les valeurs supérieures ou égales à 5\%)

La valeur support, illustrée en [7] ci-dessus et exprimée quasi-exclusivement par dans, apparaît de même $75 \%$ en tête contre $25 \%$ en queue.

Sur l'ensemble du tableau 4, on remarque que seulement deux valeurs ('quantification' et 'support') ont une nette préférence pour la position tête. Pour ces deux valeurs, nous observons des fréquences moins importantes, ce qui nous amène à émettre des réserves sur ces résultats. Néanmoins, nous pouvons retenir l'idée que la position tête puisse être préférentiellement corrélée à l'expression de valeurs moins prototypiques qui s'écartent assez nettement de la valeur spatiale. Pour toutes les autres valeurs, la proportion des occurrences en tête varie grosso-modo entre 20 et $30 \%$.

Ce premier 'filtrage' sémantique des données a mis en évidence la grande variété des valeurs pouvant être exprimées par les SP en sur et dans. Nous avons vu que les valeurs 'espace' et 'notionnel' sont quantitativement les plus représentées, et que parmi les 'notionnels', la 
'localisation' (ou espace métaphorique) est la valeur la plus importante. Nous avons aussi noté l'existence de valeurs spécifiques à chaque préposition : 'à propos' et 'quantification' pour sur, et 'support' pour dans. Ces deux dernières valeurs sont les seules à présenter une préférence nette pour la position tête.

\section{Corrélations entre fonction et position}

Qu'en est-il de la fonction? Il est avéré que les SP peuvent endosser des fonctions d'argument (insister sur les problèmes), d'adjoint (marcher sur la plage), ils peuvent modifier des têtes nominales (polémique sur les affaires), adjectivales (contrarié dans ses projets), ou prépositionnelles (jusque dans la foule) à différents niveaux de structure de l'énoncé (cf. Melis 2003, Leeman 2008, Leeman \& Vaguer 2006). Si la position initiale des SP est souvent corrélée à une fonction-extra-prédicative (portée sur la phrase ou au-delà), les SP ne sont pas pour autant interprétés 'sémantiquement' comme exophrastiques ${ }^{2}$. Bien souvent, ils participent indépendamment de leur fonction extra-prédicative à la construction du sens référentiel de l'énoncé ou véhiculent une information essentielle. Il n'y a donc pas de correspondance bi-univoque entre les notions syntaxiques intra vs. extra-prédicatifs et leur corréla sémantique endo vs. exophrastiques.

En s'appuyant sur la classification établie dans (Carter-Thomas \& Sarda sous presse), et Carlier \& Sarda (2010), nous avons classé les SP sur des bases à la fois lexico-syntaxiques et pragmatiques. L'existence d'une zone floue entre argument et adjoint est légion, notamment en ce qui concerne les SP locatifs. Pour tenter de saisir cette distinction, nous avons cherché à définir de façon détaillée différents degrés de dépendance vis-à-vis du prédicat ou de la zone prédicative. Nous avons ainsi distingué quatre degrés de dépendance dans le statut d'argument et trois degrés dans le statut d'adjoint.

\begin{tabular}{|l|l|l|l|l|l|l|l|}
\hline Prédication & $\begin{array}{l}\text { Requis \& } \\
\text { régis }\end{array}$ & Régis & Requis & Collocation & $\begin{array}{l}\text { Référen- } \\
\text { tiellement } \\
\text { requis }\end{array}$ & $\begin{array}{l}\text { Information- } \\
\text { nellement } \\
\text { requis }\end{array}$ & périphérique \\
\cline { 2 - 6 } & Arguments & Adjoints obligatoires & 'Optionnels' \\
\cline { 3 - 4 } & \multicolumn{2}{|l}{ Adjoints } \\
\hline
\end{tabular}

\footnotetext{
${ }^{2}$ Guimier (1996) distingue les adverbes endophrastiques et exophrastiques: « les premiers sont, d'un point de vue sémantique, des constituants internes à la phrase qui... participent à la construction du sens référentiel de la phrase. ... Les adverbes exophrastiques sont des constituants externes à la phrase... Ils ne participent pas à la construction de son sens référentiel, mais représentent des traces de l'intervention du locuteur » p.6.
} 
Tableau 5 : Dégrès de dépendance dans les statuts d'argument et d'adjoint

La définition des quatre degrés de dépendance des arguments repose sur la classification de Lazard (1996) basées sur les différentes combinaisons possibles des contraintes de présence, de forme et de position.

Les arguments requis et régis sont les plus intégrés. Leur présence est obligatoire et leur forme est contrainte. Ils sont illustrés en [12-13] ci-dessous :

[12] Une autre critique porte sur la survalorisation des effets bénéfiques attribués aux moyens de circulation et de télécommunications.

[13] La réponse aux difficultés réside pourtant dans la capacité à anticiper de l'Etat, de la profession et des entreprises.

Les arguments régis mais pas nécessairement requis. Ils peuvent ne pas être réalisés et rester latents (cf. la notion d'instanciation nulle, (Fillmore Ch. \& Kay P. 1996)). En revanche, si le SP est réalisé, le choix de la préposition est sélectionné par le verbe et participe parfois à activer une de ses facettes spécifiques.

[14] Dans les années 80, une filiale, le Crédit lyonnais Bank Nederland, se lance dans le financement de films indépendants américains.

[15] Dans tous les cas, le souci de ménager la production l'emporte sur les autres considérations.

[16] « Le conseil de Nice peut échouer, mais il n'échouera pas sur un différend franco-allemand. »

Les arguments requis et non régis viennent en troisième position. Leur présence est inscrite dans la structure argumentale du verbe et leur forme est libre. Cependant, comme dans le cas précédent, ces arguments peuvent aussi ne pas être réalisés et rester latents. Dans les exemples (17-18), un argument locatif est requis : emmemer quelque chose quelque part, se passer quelque part. La forme d'expression n'est pas contrainte comme en atteste la variation possible des prépositions et en dernier ressort, même si ce constituant est inscrit dans la structure du verbe, il peut rester latent, tout du moins avec le verbe emmener [17'].

[17] Ils les emmènent "dans une ville très sale " // au fond d'une cours, sur une colline etc.

[18] Et ça se passe dans l'Union européenne // à Paris, chez son oncle, près des Invalides etc.

[17'] Ils les emmènent. 
Les arguments des constructions figées ou collocationnelles. Les SP participent pleinement au sens de la construction qui ne résulte pas stricto-sensu d'un mécanisme compositionnel.

[19] Le Corbusier revient souvent dans la conversation.

[20] MOLDAVIE : le parlement est dans l'incapacité d'élire le président.

[21] L'Arabie saoudite étant quasi organiquement liée aux Etats-Unis et (...), les propos de ses dirigeants en disent long sur l'état d'esprit qui prévaut dans la région.

[22] Les préfets, répugnant à sanctionner les infractions par crainte de manifestations violentes, ferment pudiquement les yeux sur les entorses à la légalité.

[23] Issu d'une des quatre grandes familles qui gouvernent Odienné, Zakaria Touré connaît son monde sur le bout des doigts.

[24] Car les employés en ont gros sur le coeur.

La notion d'optionalité du SP est au cœur des difficultés que l'on rencontre en cherchant à établir la distinction entre argument et adjoint. Selon Dik (1997) et beaucoup d'autres

... a satellite can be left out without affecting the grammaticality or the meaning of the remaining construction, whereas living out an argument will either render the remainder ungrammatical or change its semantics (Dik 1997 : 89).

Or nous avons vu que la notion d'instanciation nulle est un phénomène fréquent qui n'affecte pas la grammaticalité. En effet, les procès décrits en [14-16] pourraient être réduits à la simple expression de la relation sujet-verbe comme en [26-27] ci-dessous :

[25] Dans les années 80, une filiale, le Crédit lyonnais Bank Nederland, se lance.

[26] Dans tous les cas, le souci de ménager la production l'emporte.

[27] « Le conseil de Nice peut échouer, mais il n'échouera pas.

Par ailleurs, le changement sémantique constaté entre [14] et [25], [15] et [26], et [16] et [27] n'est pas plus important que celui que peut entrainer la suppression d'un constituant identifié comme un adjoint. Les changements sémantiques constatés lors de la suppression d'un argument touchent en général l'aktionsart et les rôles thématiques affectés aux arguments restants. Tandis que la suppression d'un adjoint peut entraîner des problèmes de référence comme en [28], [29] et [30] où des problèmes de pertinence comme en [31], [32] et [33].

[28] a. Sur les quelque 370 sociétés mixtes installées à Cuba, une seule vient de Russie.

b. \#Une seule vient de Russie.

[29] a. Dans cet établissement, où sont nés en 1998 les premiers comités anti-Allègre, l'ensemble des enseignants dénonce la multiplication des violences.

b. \#L'ensemble des enseignants dénonce la multiplication des violences. 
[30] a. Un parfum sucré de tarte aux quetsches flotte dans le cloître, mêlé à l'odeur de la cire.

b. \#Un parfum sucré de tarte aux quetsches flotte, mêlé à l'odeur de la cire.

[31] a. Le Parlement syrien examine depuis quelques mois des projets de loi sur la création d'une Bourse et de banques à capitaux partiellement privés, dans l'objectif de réformer le secteur bancaire entièrement nationalisé en Syrie depuis 1963.

b. \# Le Parlement syrien examine depuis quelques mois des projets de loi sur la création d'une Bourse et de banques à capitaux partiellement privés.

[32] a. Ce résultat, annoncé mercredi 29 novembre, est surtout un signal important dans le marché de l'équipement des villes.

b. \# Ce résultat, annoncé mercredi 29 novembre, est surtout un signal important.

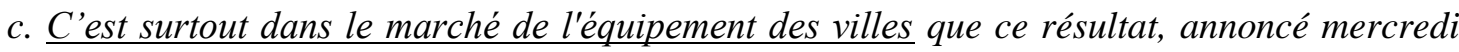
29 novembre, est un signal important.

[33] a. Ce risque existe -t-il aujourd'hui dans tous les pays de l'Union européenne?

b. \#Ce risque existe-t-il aujourd'hui?

c. \#Ce risque existe-t-il?

En somme, on constate que certains arguments peuvent être aisément omis alors que certains adjoints doivent être obligatoirement présents (cf. Goldberg \& Ackerman 2001). Parmi ces adjoints difficilement supprimables, nous avons distingués ceux qui sont reférentiellement requis et ceux qui sont informationnellement requis.

Les adjoints référentiellement requis introduisent un domaine de référence essentiel à l'identification d'un référent dans la proposition. C'est le cas dans l'exemple [28], où la présence du SP en tête de phrase est nécessaire pour le calcul de la référence du SN 'une seule', sujet de la proposition. Les référents dénotés sont liés par une relation méréologique du type membre-collection (cf. Vieu \& Aurnague 2007). De même, la référence du SN 'l'ensemble des enseignants' en [29] est définie par rapport au domaine de référence établi par le SP antéposé, ce qui permet de reconstruire le SN génitif 'L'ensemble des enseignants de cet établissement'. En [30], on a une construction existentielle avec un sujet indéfini. Si l'on supprime le SP locatif comme en [30b], le résultat est bizarre, ce qui met en évidence le rôle crucial du SP locatif 'dans le cloître' dans l'ancrage référentiel du sujet indéfini (cf. Carlier \& Sarda 2010) ${ }^{3}$.

\footnotetext{
${ }^{3}$ Dans (Carlier \& Sarda 2010), on considère que c'est la construction existentielle elle-même qui sélectionne un SP locatif de ce type. Il est dans ce cas considéré comme argument de la construction.
} 
Les adjoints informationnellement requis véhiculent une information cruciale qui, si elle était supprimée, aurait un impact sur le bien fondé de l'acte de communication. En [31], le SP postposé exprime le but et justifie les événements décrits par la proposition qui précède (examiner les projets de loi dans l'objectif de reformer). Dans l'exemple [32], le SP adverbial vient modifier l'adjectif important, mais comme on peut le voir en [32c], il peut être focalisé. Sa suppression entraînerait la perte d'une information cruciale du message. Enfin dans l'exemple [33], on observe que le but du message n'est pas de questionner sur l'existence du risque en soi [33c], mais bien de localiser, à la fois dans l'espace et dans le temps , l'existence du risque.

Les adjoints périphériques, enfin, sont ceux ne sont pas impliqués dans l'identification des référents des arguments de la proposition, et qui véhiculent une information plutôt d'arrière plan (setting) de sorte que leur suppression ne viole pas la maxime de quantité (Grice 1975) et résulte en un message qui reste informatif. Ils sont illustrés en [34-37] ci-dessous :

[34] Dans l'Hémicycle, les parlementaires avaient dénoncé la procédure expéditive des ordonnances, les communistes et les députés chevènementistes $(M D C)$ s'alliant à la droite pour voter contre le texte.

[35] Dans tous les cas, le souci de ménager la production l'emporte sur les autres considérations.

[36] Sur le plan pratique, le système Thinkwave permet aux professeurs de gagner du temps : il facilite l'organisation des tâches quotidiennes, simplifie le traitement des notes et supprime une partie de la paperasserie.

[37] Sur les neuf dernières années, les meilleurs skieurs français ont retiré de ce fonds une moyenne annuelle de 7,6 millions de francs

La gradation que nous avons établie entre les différents statuts d'argument et d'adjoint (cf. tableau 5) reflète différentes contraintes de dépendance vis-à-vis du prédicat, de la prédication ou de la proposition. Il s'agit de contraintes lexico-syntaxiques (règles de souscatégorisation), de contraintes sémantico-référentielles et de contraintes pragmatiques. Nous avons considéré que les adjoints référentiellement requis sont plus dépendants que les adjoints informationnellement requis dans la mesure où leur suppression nuit véritablement à l'identification des référents dénotés dans la partie restante de l'énoncé. Les adjoints informationnellement requis véhiculent une information saillante. Leur suppression rend le message bizarre parce que peu informatif mais, sans anomalie grammaticale.

Dik distinguent les constituants extra-phrastiques (extra-clausal constituents) des constituants phrastiques par le fait qu'ils sont non essentiels, par le fait qu'ils sont séparés de la phrase par une pause, et par le fait qu'ils ne sont pas sensibles aux règles grammaticales opérant dans la 
phrase. Il souligne cependant que ces constituants extra-phrastiques peuvent être liés à la phrase par des règles impliquant des phénomènes de co-référence, de parallélisme ou de contraste (cf. Dik 1997, part II : 381). C'est précisément ce type de liens, qui d'un point de vue sémantico-pragmatique, imposent une contrainte de présence. Dans l'analyse que nous avons menée, nous avons été très attentives à ces différents liens possibles entre les constituants du SP et ceux de la zone prédicative, qu'ils soient de nature lexico-syntaxique, ou sémantico-pragmatique. Il était en effet crucial de tenir compte de la complexité de ces liens pour pouvoir comparer l'effet de la position en tête et en queue des SP.

Le tableau 6 ci-dessous permet de vérifier une tendance générale qui associe les fonctions d'adjoint et la position tête, et les fonctions d'argument à la position postverbale. Plus précisément, on observe parmi les adjoints, que les périphériques sont plus fréquents en tête (62\%), de même que les adjoints référentiellement requis (85\%). Ce dernier résultat est en accord avec l'idée que la référence se construit au fil du texte et que la suppression d'éléments en amont est nuisible à l'interprétation de ce qui reste en aval. De ce point de vue, il est logique de trouver plus d'adjoints référentiellement requis en tête qu'en queue. En revanche, les adjoints informationnellement requis sont plus fréquents en queue $(60 \%)$, ce qui est aussi conforme à l'idée que l'information nouvelle (saillante) apparaît naturellement dans la zone rhématique (post-verbale pour le français). Quant aux arguments, ils sont très fortement associés à la position queue, entre $80 \%$ pour les arguments requis, jusqu'à $100 \%$ pour les collocations.

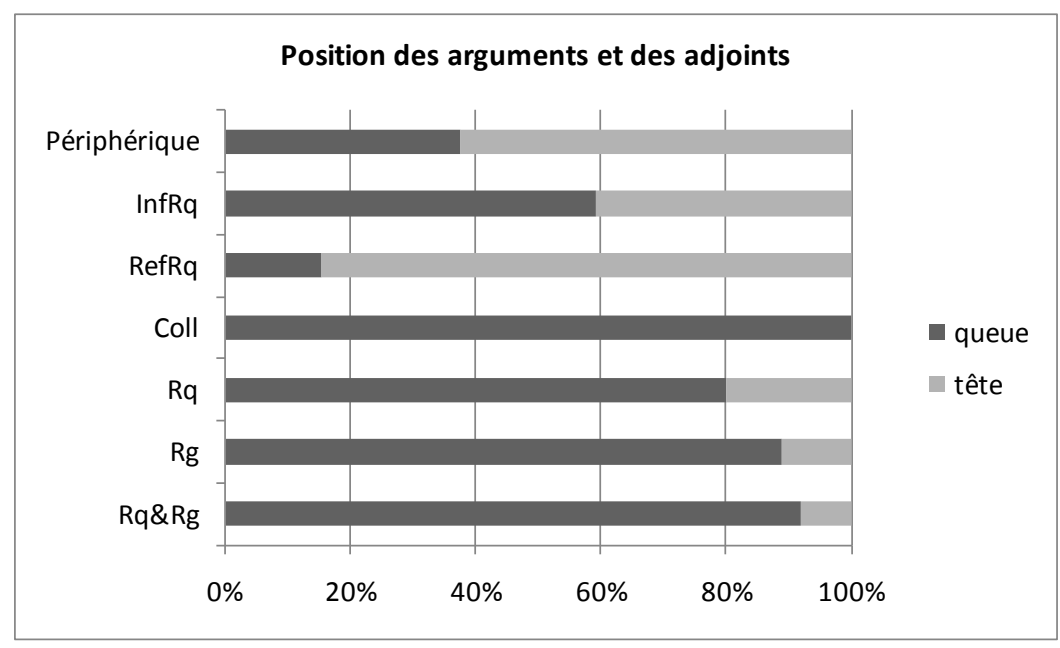

Tableau 6 : Corrélations entre position et fonction des SP 
De façon plus détaillée, on peut observer, dans le tableau 7 ci-dessous, quelles sont les valeurs sémantiques préférentielles associées aux fonctions d'adjoint ou d'argument selon la position.

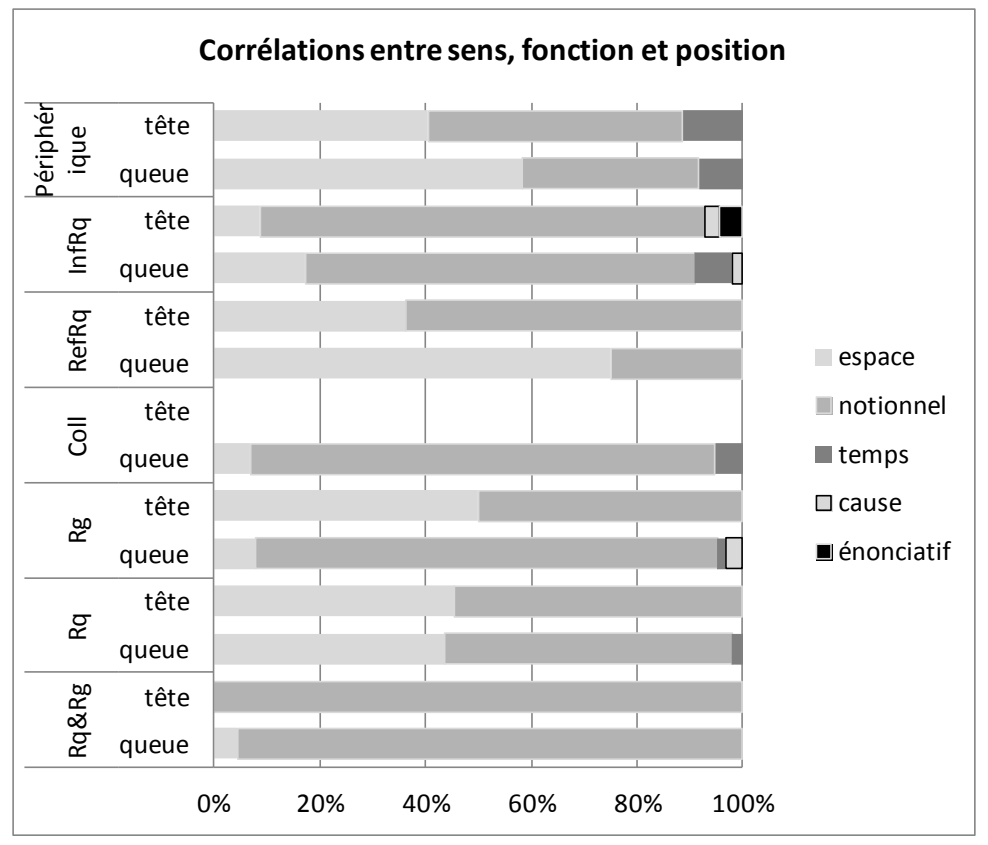

Tableau 7 : Corrélations entre sens, fonction et position

Nous avons vu que les SP proprement périphériques sont plus fréquents en tête (62\%) qu'en queue (38\%) (cf. Tableau 6). Lorsqu'ils apparaissent en tête, on observe une légère prévalence des emplois notionnels (48\%) pour 41\% d'emplois spatiaux et $11 \%$ temporels. En fin de phrase, en revanche ce sont les emplois spatiaux qui prévalent (58\%) sur les notionnels $(33 \%)$, et les temporels $(8 \%)$.

Pour les adjoints requis informationnellement (qui prévalent en queue), on ne note pas de différences importantes de valeurs sémantiques qui seraient plus fréquentes dans l'une ou l'autre position. Pour les adjoints requis référentiellement (qui prévalent en tête), on note en revanche une prévalence des emplois notionnels en tête $(64 \%)$ contre $36 \%$ d'emplois spatiaux, et inversement, une prévalence des emplois spatiaux en queue (75\%) contre $25 \%$ de notionnels. Cette distribution correspond aux deux principales constructions identifiées, à savoir la quantification en tête, et le locatif en queue dans les phrases à sujet indéfini. Ces constructions sont illustrées par les exemples repris ci-dessous :

[38] Sur les quelque 370 sociétés mixtes installées à Cuba, une seule vient de Russie.

[39] Un parfum sucré de tarte aux quetsches flotte dans le cloître, mêlé à l'odeur de la cire. 
De façon plus synthétique, le tableau 8 rend compte (en $\%)^{4}$ des préférences observées entre sens et fonction. On a déjà relevé le fait que $62 \%$ des occurrences des deux prépositions confondues ont une fonction d'adjoint. On peut ajouter à cette observation que pour toutes les valeurs sémantiques, la fonction d'adjoint prévaut: elle est de $100 \%$ pour la valeur énonciative, $91 \%$ pour la valeur temporelle, $72 \%$ pour la valeur spatiale, $67 \%$ pour la valeur causale et $56 \%$ pour la valeur notionnelle. La valeur notionnelle est donc celle qui est a contrario la plus présente dans la fonction d'argument (44\%).

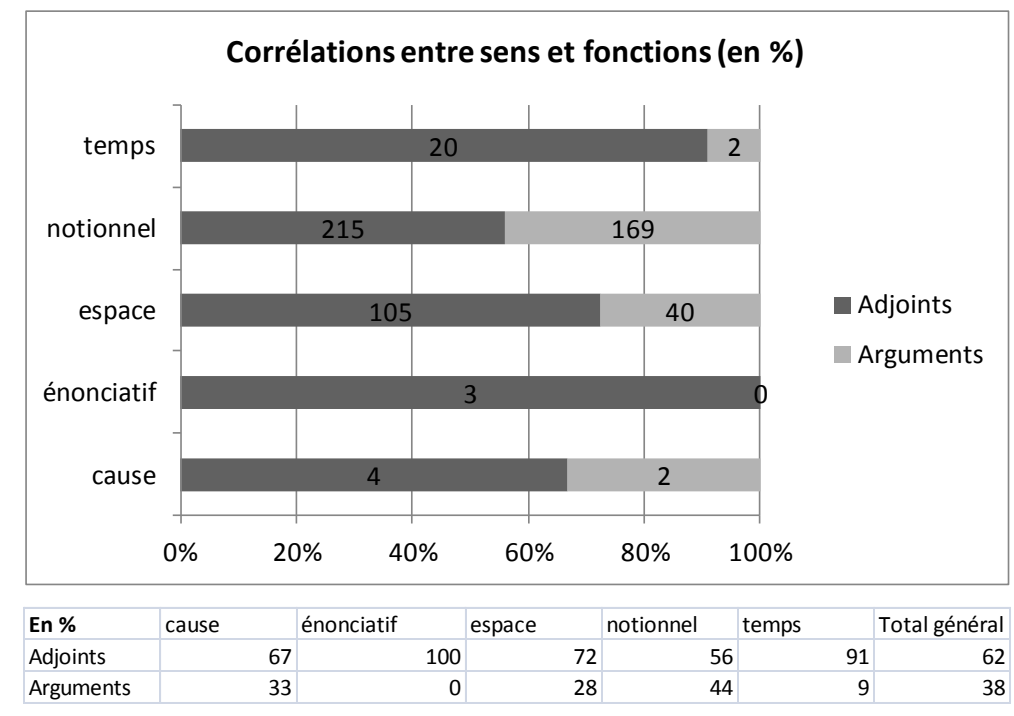

Tableau 8 : Tableau synthétique des corrélations entre sens et fonction

Sur la base de cette description, nous allons examiner dans la troisième partie les fonctions textuelles que peuvent prendre les SP en sur et dans. La question initiale était de discerner ce qui, de leur sens, de leur fonction ou de leur position, leur permettait de remplir de telles fonctions à l'échelle du discours. Le point méthodologique fondamental sur lequel nous voulons insister est que si nous voulons étudier l'impact de la position sur le rôle discursif des $\mathrm{SP}$, nous devons comparer des SP ayant le même statut et la même autonomie vis-à-vis du prédicat. Nous avons pu mettre en évidence que certaines valeurs sémantiques sont plus intrinsèquement «circonstancielles » et donc moins sensibles à la position. Par exemple, le temps reste intrinsèquement circonstanciel en tête comme en queue (i.e. la fonction d'adjoint prévaut). La valeur notionnelle est au contraire extrêmement sensible à la position : en tête, on observe $91 \%$ d'adjoints pour seulement $39 \%$ en queue. De même pour la valeur spatiale, mais de façon moins marquée, on observe $86 \%$ d'adjoints en tête pour $62 \%$ en queue.

\footnotetext{
${ }^{4}$ Le nombre exact d'occurrences est indiqué sur les barres du graphe.
} 


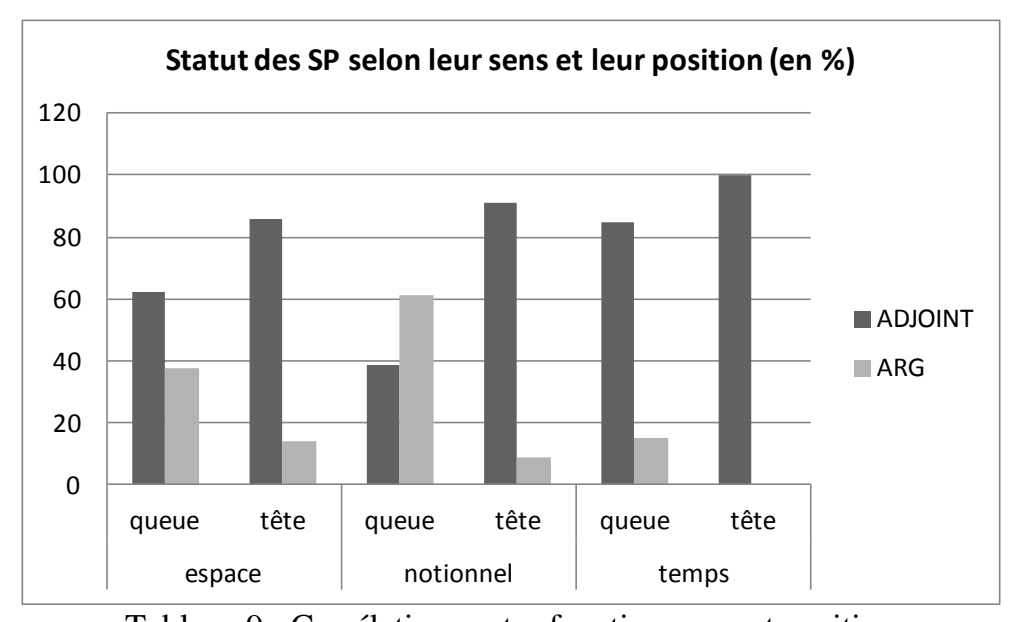

Tableau 9 : Corrélations entre fonction, sens et position

La question sur laquelle nous nous concentrons est la suivante : à statut égal, quels sont les effets spécifiques du positionnement en tête que nous n'observons pas en queue ?

\section{Fonctions textuelles des SP}

Dans cette troisième partie, nous examinons les fonctions organisatrices des SP au niveau textuel et l'incidence de la position sur la mise en œuvre de ces fonctions.

\subsection{Structuration cadrative :}

La portée d'un constituant initial au-delà de sa phrase d'accueil est définie par Charolles (1997) comme un outil de structuration à l'échelle du discours. De même que les connecteurs établissent des liens avec le co-texte qui précède, de même les adverbiaux en position initiale, adverbiaux dits cadratifs, établissent des liens avec le co-texte qui suit. Ils créent des blocs d'informations homogènes vis-à-vis du critère qu'ils fixent. L'exemple 40 en est une illustration :

[40] Pour cet adolescent de dix-sept ans, sans emploi, la candidature de Simone Gbagbo, l'épouse du chef de l'Etat, est une provocation. "Abobo est un fief du RDR, dit-il. Elle ne peut gagner ici qu'en nous excluant. » $P_{0}$ [Sur l'ensemble du pays, l'élection s'est déroulée dans des conditions contrastées. [Dans le Sud, le scrutin n'a été entaché d'aucun incident, même si la participation a été faible]. [Dans le Nord, le vote a été perturbé par les partisans d'Alassane Ouattara au point qu'il n'a pas eu lieu dans cinq des treize départements septentrionaux. [Dans plusieurs bureaux de vote, le matériel électoral a été brûlé]. [Ailleurs, les fonctionnaires en charge du scrutin ont été intimidés et se sont réfugiés à la préfecture ou à la gendarmerie].[...]]]. 
Dans cet exemple, un premier SP (Sur l'ensemble du pays) ouvre un cadre à l'intérieur duquel prennent place deux nouveaux cadres dépendants (Dans le sud/ Dans le nord). Le texte progresse par une description des événements se produisant dans le Nord, et les informations sont réparties dans deux nouveaux sous-cadres (Dans plusieurs bureaux de vote - Ailleurs). On observe un emboitement hiérarchique des différents espaces.

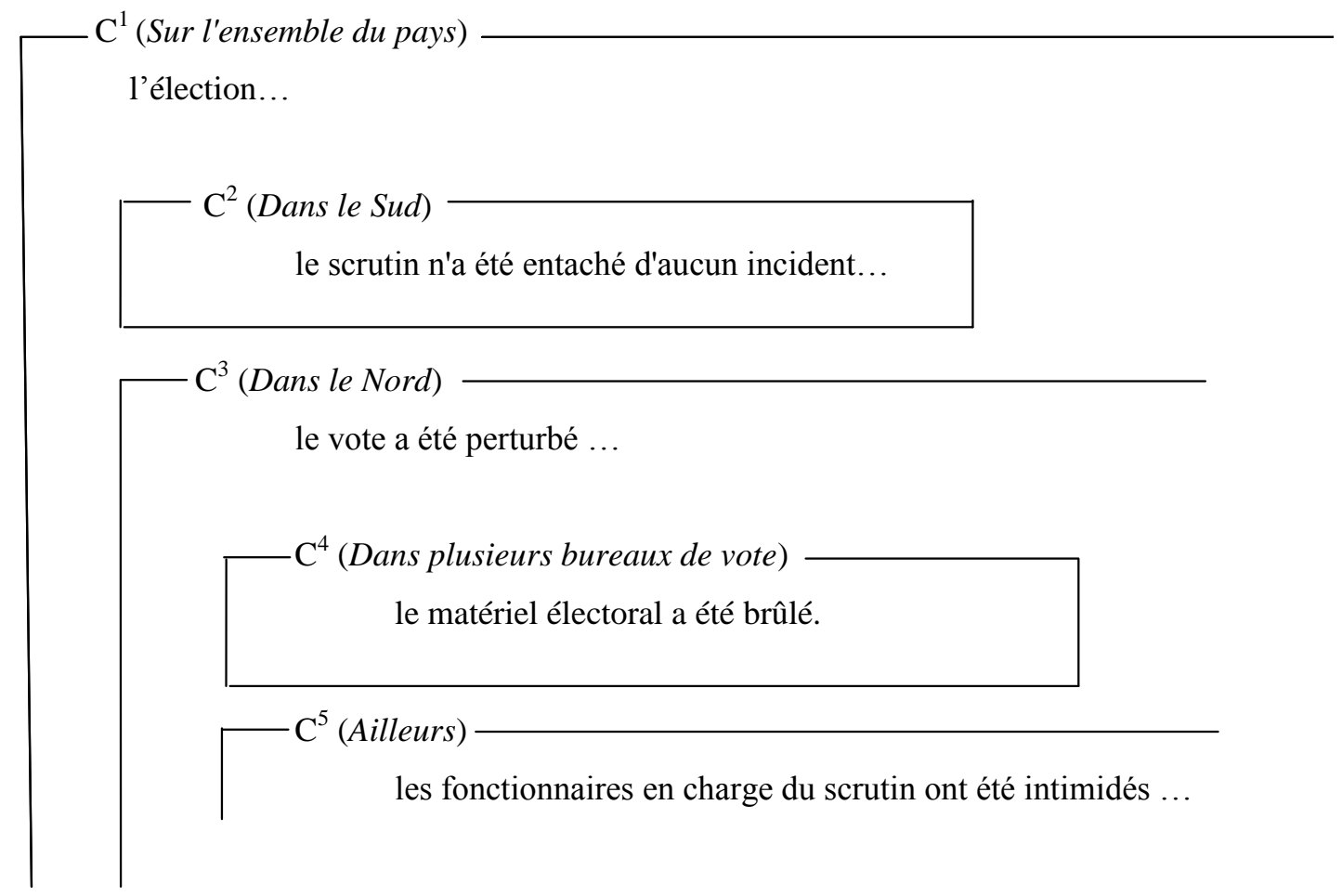

Cette structure d'emboitement hiérarchique des différents espaces est soutenue par le placement des SP (spatiaux) à l'initiale des phrases. Elle donne un accès à l'information en adoptant ce qu'Enkvist (1987), ou Virtanen (1992) nomment une 'stratégie textuelle locative'. Avec cette stratégie, l'adverbial spatial participe à l'inscription du topique ou thème sur un fond (ou 'ground' cf. Tamly 2000). Ainsi, ce 'à propos' de quoi on parle n'est pas des élections, mais des élections sur l'ensemble du pays. Ce qu'apporte l'information nouvelle dans la zone rhématique, c'est que l'événement, ancré spatialement (les élections sur l'ensemble du pays), s'est déroulé dans des conditions contrastées. L'attention porte donc sur l'aspect contrasté du déroulement des élections, et ce contraste peut naturellement être développé à la suite du texte. La phrase $\mathrm{P}_{0}$ fonctionne en effet comme phrase amorce d'une structure énumérative (cf. Ho-Dac \& al. 2010) dont les items sont également introduits par des SP spatiaux (dans les sud/ dans le nord) désignant des sous-parties de l'espace de référence introduit en $\mathrm{P}_{0}$. L'idée sous-jacente à cette description est que le placement en position initiale des SP ne se justifie pas à l'échelle de la phrase mais bien, à l'échelle du 18 
discours. A l'échelle de la phrase le placement en queue est syntaxiquement possible comme en atteste la manipulation de [40] en [40'] ci-dessous :

[40'] Pour cet adolescent de dix-sept ans, sans emploi, la candidature de Simone Gbagbo, l'épouse du chef de l'Etat, est une provocation. "Abobo est un fief du RDR, dit-il. Elle ne peut gagner ici qu'en nous excluant. » $P_{0} L^{\prime}$ élection s'est déroulée dans des conditions contrastées sur l'ensemble du pays. $P_{1}$ Le scrutin n'a été entaché d'aucun incident, dans le Sud, $P_{2}$ même si la participation a été faible. $P_{3}$ Le vote a été perturbé par les partisans d'Alassane Ouattara $P_{4}$ au point qu'il n'a pas eu lieu dans cinq des treize départements septentrionaux, dans le Nord. $P_{5}$ Le matériel électoral a été brûlé dans plusieurs bureaux de vote. $P_{6}$ Les fonctionnaires en charge du scrutin ont été intimidés ailleurs, et $P_{7}$ se sont réfugiés à la préfecture ou à la gendarmerie.

Notons que si le placement du SP (sur l'ensemble du pays) en fin de phrase est syntaxiquement possible, il peut paraître, dans ce cas, pragmatiquement incongru parce que le positionnement en fin de phrase le place de fait dans la zone rhématique ${ }^{5}$. Or le texte ne progresse pas à propos de 'l'ensemble du pays', mais à propos d'un événement (un scrutin sans incident) identifié par sa localisation 'dans le sud'. Notons également que le placement du SP (sur l'ensemble du pays) en fin de phrase est alors susceptible de créer un hiatus sémantique avec les constituants en fin de phrase : 'les conditions contrastées' évoquent plus immédiatement les entités qui sont contrastées ( $\mathrm{p}$ dans le sud et q dans le nord) plutôt que l'ensemble dans lequel elles sont saisies (l'ensemble du pays). Cela n'empêche pas néanmoins que ce SP manipulé et placé en fin de phrase ait une portée sur la phrase qui suit, mais il apparaît que c'est par un principe économique de conservation par défaut des informations spatio-temporelles, plutôt que par un principe constructionnel comme c'est le cas lorsqu'il est en position initiale.

En lisant cet exemple manipulé, on se heurte en effet rapidement à un problème de structure. On interprète par défaut $\mathrm{P}_{1}$ comme dépeignant un événement relatif à l'ensemble du pays jusqu'à ce qu'on rencontre en fin de phrase le deuxième SP spatial (dans le sud) qui en désigne une sous-partie. La compatibilité de la partie (le sud) avec le tout entraîne une simple

\footnotetext{
${ }^{5}$ On pourrait tenter de le séparer par une virgule, mais le résultat n'est pas plus convaincant. (L'élection s'est déroulée dans des conditions contrastées, sur l'ensemble du pays).
} 
accommodation $^{6}$ qui nécessite néanmoins un retraitement de l'information véhiculée par P1 (qui reste peu couteux du fait que la phrase est courte). Les difficultés réelles commencent à la lecture de $\mathrm{P}_{3}$, et sont d'ordre sémantique. Du fait que le SP spatial dans le sud est toujours actif par défaut, on est enclin à interpréter le nouvel événement introduit par $\mathrm{P}_{3}$ 'un vote perturbé' dans sa portée. Mais on éprouve des difficultés de conceptualisation à ancrer en un lieu unique (dans le sud) la manifestation d'un événement (l'élection, le vote, ou le scrutin) individualisée par des propriétés contradictoires (sans incident vs. pertubé). Si un événement a des manifestations différentes au même moment il faut que ce soit dans des lieux différents ${ }^{7}$, d'où le choix stratégique du placement de l'adverbial 'dans le nord' en tête, dans la version originale, qui permet ainsi d'éviter un ancrage erroné de $\mathrm{P}_{3}$ 'dans le sud'.

A travers l'analyse et la manipulation de cet exemple, nous avons tenté de montrer que le placement des SP en fin de phrase n'entraîne (le plus souvent) aucune agrammaticalité au niveau de la phrase, mais produit de grandes difficultés sémantiques et pragmatiques au niveau du texte parce que les SP ne peuvent alors plus jouer à temps leur fonction de signalisation. L’information qu'ils véhiculent arrive trop tard et un retraitement est nécessaire. On peut considérer de fait que la position du SP en tête de phrase répond à un principe d'économie pour orienter et guider l'interprétation de la ou des phrase(s) qui suit ou suivent en évitant de surcroît qu'elle(s) ne soi(en)t interprétée(s) selon d'autres critères préalablement introduits. Le rôle du placement du SP en position initiale est donc double : il vise tant à fournir un critère d'interprétation à la phrase qui suit qu'à bloquer la portée d'un critère énoncé en amont. Il est intimement lié à la linéarité du texte et fonctionne à la manière d'un panneau de signalisation pour le lecteur indiquant tant la borne finale d'un cadre antérieur (ou d'une information qui serait maintenue par défaut dans l'interprétation de la suite du texte) que la borne initiale d'un nouveau cadre. On peut donc le caractériser comme une balise charnière orientant l'interprétation et délimitant des segments. Mais il faut bien reconnaitre

\footnotetext{
${ }^{6}$ (cf. van der Sandt 1992). Notons au passage que l'introduction d'un espace disjoint (par exemple, en Europe) à la place de la sous partie (le sud) aurait en revanche entraîné un conflit.

${ }^{7}$ Dans l'ontologie des entités spatio-temporelles définie par Zemach (1979), le vote appartient aux entités qui sont caractérisées par le fait d'être continues dans la dimension spatiale et discontinues dans la dimension temporelle. Comme d'autres entités du même type la crise bancaire, la révolution industrielle, si le vote se produit à un temps $\mathrm{t} 1$, dans plusieurs lieux disjoints $(11,12)$, il s'agit du même vote. Si dans un même lieu (11), le vote a lieu à des moments distincts ( $\mathrm{t} 1$ et $\mathrm{t} 2)$ il s'agit en revanche de deux votes $\left(1^{\mathrm{er}}\right.$ tour, $2^{\text {ème }}$ tour par exemple). Et on peut donc ajouter que si le vote se manifeste différemment au même moment, ce doit forcément être dans des lieux différents.
} 
que ces propriétés ne sont pas celles des SP, mais bien celles de la position initiale elle-même car les SP, à statut égal (i.e. adjoint périphériques) ne guident pas l'interprétation de la même façon lorsqu'ils sont en tête ou lorsqu'ils sont en queue.

Il faut aussi insister sur le fait que cette place (slot) à l'initiale de phrase se caractérise par le réseau qu'elle tisse avec d'autres places initiales de phrase comme dans les exemples [41-43] ci-dessous.

[41] Pour empêcher que le progrès social apparaisse comme un obstacle au progrès économique et que se développent des processus de spirale sociale descendante, seule une certaine mondialisation de la législation sociale peut tenir tête à la mondialisation de l'économie. Sur le plan des idées, il est donc plus urgent que jamais que les valeurs et principes de base de l'OIT soient rappelés sans relâche, précisés, enseignés et autant médiatisés que les valeurs économiques d'efficacité, de puissance ou de compétitivité. Sur le plan opérationnel, il faudrait que les principales normes de l'OIT-et en particulier celles relatives à des droits sociaux fondamentaux tels que la liberté syndicale ou le droit de négociation collective-deviennent beaucoup plus contraignantes.

[42] Dans certains cas, le traité renvoie à plus tard la décision de passage à la majorité qualifiée, sans certitude quant au résultat final ; dans d'autres, le renoncement à l'unanimité est assorti de conditions restrictives.

[43] Sur la corse, M. Chirac a mis en garde le gouvernement. A ses yeux, les transferts de compétences législative et réglementaire supposent une révision constitutionnelle soumise au référendum. Sur l'Algérie, le président a renvoyé dos à dos les auteurs des "atrocités » commises des deux côtés.

On peut imaginer une macro-construction à l'échelle du texte qui se fonde en premier lieu sur les liens structurels entre les constituants initiaux (macro-structures de listes articulant des parallélismes ou des contrastes). La portée sur plusieurs phrases est selon nous une propriété secondaire (une potentialité qui n'est pas toujours réalisée) des SP initiaux dit cadratifs, alors que la possibilité de la relation entre plusieurs autres SP en position initiale fonde le rôle structurant de la position. C'est-à-dire que même si l'introduction d'un premier adverbial en position initiale n'est pas suivie par l'apparition d'un autre, ou de plusieurs autres, le fait de délimiter un domaine, un espace, une temporalité, un point de vue etc. créer implicitement en creux le domaine complémentaire à celui qui est ainsi posé, de la même manière qu'en traçant une forme convexe sur une page blanche, on délimite du même coup l'espace concave à l'entour. 
Le corpus étudié (Le Monde), nous avons trouvé 12\% de SP (en sur ou dans) ayant une portée sur plusieurs phrases. Toutes ces occurrences ont une fonction d'adjoint et sont en position initiale. Elles représentent environ $38 \%$ des SP en tête. Il est certain que d'un point de vue quantitatif, ce corpus n'exploite pas pleinement la potentialité des SP cadratifs. Cela peut-être mis sur le compte du genre de la Presse journalistique qui est très contraint : les articles sont en général courts et l'information peu développée et peu argumentée. Nous avons en effet beaucoup d'exemples où les SP en tête seraient de bons cadres potentiels mais ne sont finalement pas exploités, probablement pour des contraintes d'espace. De plus les contraintes de rapidité de rédaction aboutissent souvent à des textes non ou mal planifiés. Le genre est donc un facteur déterminant sur la possibilité d'exploitation des cadres. Certains travaux (HoDac 2007, Virtanen 1992) ont pu montrer que ce potentiel est pleinement à l'œuvre dans les textes expositifs, descriptifs, et littéraires (tels qu'on les trouve dans les articles scientifiques, les manuels scolaires, les romans).

Nous avons jusqu'ici veillé à comparer des SP de même statut syntaxique en tête et en queue. Nous avons donc considéré des SP qui sont, dans les deux positions, syntaxiquement indépendants de la zone prédicative. Il ressort qu'aucun SP adjoint en zone finale n'a de portée au sens cadratif au-delà de la phrase. Cependant, nous avons pu observer (cf. exemple [40’]) que les informations exprimées par des SP en fin de phrase peuvent parfois être maintenues par défaut pour l'interprétation de la suite (ancrage par défaut), mais cela n'en fait pas pour autant un outil de structuration. Au contraire, la portée de ses informations est aléatoire, tributaire des associations possibles avec les informations arrivantes et pour ainsi dire «sauvage », dans la mesure où elle ne peut être maîtrisée et canalisée que par les expressions en tête de phrase qui jouent le rôle de balises. Ce qui est donc spécifique à la position initiale, c'est un principe d'économie qui permet de guider l'interprétation au fil du texte (signalisation du texte). A l'inverse, placer ces critères d'interprétation en fin de phrase peut entrainer la nécessité d'un retraitement du début des phrases qui restait par défaut dans la portée d'un critère antérieur.

La position initiale est donc cruciale dans la construction de la structure du texte. Cela ne veut pas dire que les SP en fin de phrase ne jouent aucun rôle, on peut même penser que le placement en tête libère l'espace en fin de phrase pour d'autres fonctions: dès lors que les SP apparaissent en fin de phrase, ils sont susceptibles d'être attirés dans la zone rhématique et on s'attend à ce qu'ils remplissent en effet d'autres fonctions que celle de 'signalisation'. Et de 
fait, on trouve en position finale des SP qui participent à la structuration d'une autre manière, en déclenchant des progressions dites à 'thèmes dérivés' (cf. Daneš 1970, 1974, Combettes 1983). Mais il est rare que ces SP soient des adjoints. Ils sont généralement fortement intégrés à la structure prédicative comme nous allons le voir dans la prochaine section.

\subsection{Hyperthème (ou Progression à thèmes dérivés)}

Les adjoints périphériques véhiculent des informations d'arrière-plan qui servent à l'interprétation des événements proprement dits. Nous avons vu qu'il est donc plus économique des les placer en tête pour éviter une re-conceptualisation de l'événement une fois qu'on arrive à la fin de la phrase. Cette observation est sous-tendue par l'analyse de nos données qui montraient (cf. section 2) qu'en zone finale, peu de SP sont de vrais périphériques (38\%). On trouve plus massivement dans cette zone finale les adjoints informationnellement requis $(60 \%)$, et plus rarement les adjoints référentiellement requis (15\%). Dans ces deux derniers cas, les SP sont constitutifs de l'information rhématique. A ce titre, ils peuvent à leur tout fournir (de la même façon qu'un argument) le point de départ d'un développement du texte. Sans pouvoir parler de portée au sens structurel de guidage de l'interprétation, ils peuvent avoir un autre rôle, celui d'hyper-rhème ${ }^{8}$ déclenchant des progressions à thèmes dérivés.

Nous nous référons au troisième grand type de progression thématique distingué par Daneš (1970, 1974). Pour aborder l'organisation thématique au niveau global du texte, F. Daneš a établi trois schémas représentatifs qui rendent compte de la progression thématique: la progression linéaire (le rhème de la première phrase devient le thème de la phrase suivante et ainsi de suite), la progression à thème constant (à un thème constant s'adjoignent différents rhèmes successifs) et la progression à thèmes dérivés. Dans la progression à thèmes dérivés, la progression s'organise à partir d'un hyper-thème dont différents sous-thèmes sont développés (cf. Daneš 1974 : 106-128). Ce type de configuration figure relativement souvent dans les textes scientifiques et permet d'organiser des énumérations autour d'un point de départ donné (Carter-Thomas 1999 ; 2010). Il fournit à l'énonciateur un moyen de détailler et d'expliquer une organisation arborescente.

Contrairement à la structuration cadrative qui établit un point d'ancrage commun pour rassembler des événements différents [44], la progression à thème dérivé se développe à partir d'un événement (ou une entité) unique qui est ensuite élaboré par un ensemble de sous-

\footnotetext{
${ }^{8}$ Combettes (1983: 97) parle d'hyperthème en position de rhème.
} 
événements constitutifs [45]. Combettes parle de progression à thème «éclaté » (1980: 99) très fréquente dans les descriptions et les explications :

[44] [C, p.q.r.etc.]

[45] p..........(Rhème 1)

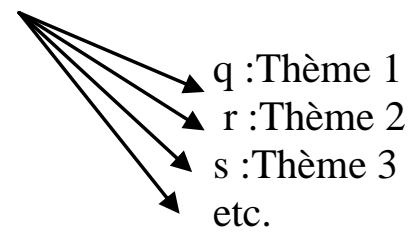

Dans les termes de Halliday (1994), on peut concevoir cette distinction entre structuration cadrative et ce type de progression thématique en montrant que l'une relève en premier lieu de la composante textuelle et l'autre de la composante idéationnelle, que l'une convoque les circonstances, l'autre les participants. Mais il ne serait pas tout à fait juste de réduire la complexité dans cette série d'oppositions. En particulier, les éléments pré-sujets en position initiale peuvent en eux-mêmes jouer au niveau de différentes composantes : interpersonnelle pour les adverbiaux énonciatifs, textuelle pour les adverbiaux organisationnels (cf. marqueurs d'intégration linéaire), ou idéationnelle pour les adverbiaux circonstanciels. Mais tous, de par le fait qu'ils apparaissent en position initiale, fonctionnent au niveau de la composante textuelle. Ils servent en premier lieu à guider l'interprétation des contenus, et peuvent cumulativement véhiculer eux-mêmes un contenu représentationnel. Les hyper-rhèmes sont quant à eux au cœur de la composante idéationnelle et s'ils participent à la cohésion du texte ils ne participent pas à la structuration du texte.

Les différences majeures entre ces deux modes de cohésion textuelle sont à la fois syntaxiques et informationnelles: les cadres, en tête de phrase, sont des adjoints périphériques, et constituent l'arrière plan des informations thématiques ou topicales (pour plus de détails sur le statut informationnel des cadres voir Charolles 2003, Sarda \& CarterThomas 2009). Les SP en fin de phrase jouant le rôle d'hyper-thème sont quant à eux syntaxiquement plus intégrés et constituent l'information rhématique qui va être élaborée dans la suite du texte. Ce dernier type de structuration exploite non pas les constituants périphériques mais les constituants majeurs du noyau de la phrase. 
Dans notre corpus, nous avons plusieurs exemples où un SP rhématique donne lieu à toute une séquence de sous-thèmes de ce type :

[46] Une série d'exercices porte sur trois documents supports. Un texte de Buffon énonce la supériorité de l'homme, être pensant, sur l'animal. Une bande dessinée de Reiser représente deux savants dans leur laboratoire, qui, l'air satisfait, passent en revue des animaux soumis à des réflexes conditionnés, puis se rendent au distributeur de café, en panne. Le troisième document est une nouvelle de Dino Buzzati, Les Souris. Un narrateur y raconte la progressive prise de pouvoir par des souris sur une maison et ses habitants. A la fin du récit, ces derniers ont été réduits en esclavage tandis que les premières sont devenues des rats noirs énormes. Ces trois documents concernent donc le rapport des hommes aux animaux, exclusivement présenté sous l'angle de la domination.

Dans cet exemple, le SN trois documents supports apparaît comme argument du verbe porter sur. Ce SN figure dans la portion rhématique de la première phrase ; il constitue l'hyperrhème des sous-thèmes développés dans les propositions qui suivent : Un texte de Buffon énonce ... ; Une bande dessinée de Reiser représente... ; Le troisième document est une nouvelle de Dino Buzzati, Les Souris ${ }^{9}$. L'énumération se clôt ensuite par la reprise du SN hyper-rhème avec un démonstratif ces trois documents. On voit dans cet exemple qu'à la différence de la structuration par les cadratifs en tête de phrase, le point du départ de l'enchainement n'est pas un 'setting' qui fournirait un critère d'interprétation ou une orientation stable pour les énoncés à venir. Il s'agit d'une entité, exprimée par un constituant intégré, qui est explicitée en sous-thèmes constituant l'à propos de la suite du texte. Comme le souligne Combettes (1983:99), «c'est la répétition d'une même structure qui permet de parler de sous-thèmes ». Il note en effet que si l'une des phrases était isolée et n'avaient pour référent le SN (trois documents supports, dans notre exemple), l'analyse serait différente. L'exemple qui suit offre une autre illustration de cette fonction d'énumération :

[47] Nommé à la tête du CEA-I, Jean-Claude Hirel avait pour mission de transformer cette holding en une entreprise industrielle pesant 46 milliards de francs de chiffres d'affaires et regroupant 56 000 salariés. Ce nouvel ensemble reposait sur quatre pôles. L'électronique de Thomsoncomprenant les semi-conducteurs du franco-italien SGS Thomson, les téléviseurs de TCE (Thomson Consumer Electronics), ainsi que l'électroménager-s'ajoutait aux activités du CEA : l'électronucléaire, avec les participations dans la Cogema et Framatome, le biomédical, via Oris

\footnotetext{
${ }^{9}$ On peut noter une rupture du parallélisme dans cette troisième phrase qui introduit le nom du document en position rhématique.
} 
et Sopha Medical, et les services informatiques à travers la CISI. Ce schéma n'a pas résisté à l'épreuve des hommes et des faits.

Il nous a semblé important de mettre en regard ce type de phénomène assez fréquent progression à thème dérivée - avec les cadres et la notion de portée parce que la limite entre les deux n'est pas si évidente. Cependant, plusieurs distinctions importantes peuvent être établies entre les deux types de structuration :

Comme nous venons de voir dans les exemples ci-dessus, les progressions thématiques s'organisent autour des constituants majeurs de la phrase. Dans (47) par exemple c'est le SN 'sur quatre pôles' en position de rhème qui fournit les thèmes des quatre phrases suivantes. Il s'agit d'un constituant essentiel sur le plan syntaxique, alors que le phénomène cadratif est strictement corrélé en français à un constituant périphérique en position initiale.

De plus, la progression thématique quel qu'elle soit, est quelque chose qui parcourt l'ensemble du texte et qui en est constitutif : en effet, il y toujours nécessairement au niveau du texte une progression thématique, qui peut certes comporter des ruptures et des nouveaux thèmes, mais qui est nécessairement présente. Or, en ce qui concerne les cadres, il s'agit d'un phénomène plus optionnel, plus éphémère, qui jalonne les contenus déployés dans le texte.

On peut ajouter à ces observations une série de remarques qui pointent encore d'autres différences entre ces deux types de structuration: La zone d'influence de chaque thème est uniquement phrastique, et plus individuelle, au sens où les liens transphrastiques sont établis à partir de la concaténation des thèmes. Les cadres peuvent quant à eux fournir une réorientation (portée) pour plusieurs phrases. Les cadres et les thèmes peuvent cohabiter, ils offrent deux types de structuration complémentaires. Les hyper-rhèmes engendrent des énumérations dont le point de départ est exprimé par un constituant intégré de l'énoncé (sujet ou objet (cf. ex trois documents). La structure de ces énumérations est peu contrainte. Dans l'exemple [46], on a vu qu'il y avait une rupture du parallélisme (Le troisième document est une nouvelle de Dino Buzzati) avec l'introduction du nom du document comme rhème alors que les deux précédents étaient introduits comme thème. En [47], il faut rechercher les entités énumérées avec plus d'attention encore, car elles sont distribuées sur une structure complexe qui contient elle-même un hyper-rhème ('les activités du CEA'). Cet exemple est représenté sous $[48]$ : 
[48] Ce nouvel ensemble reposait sur quatre pôles.

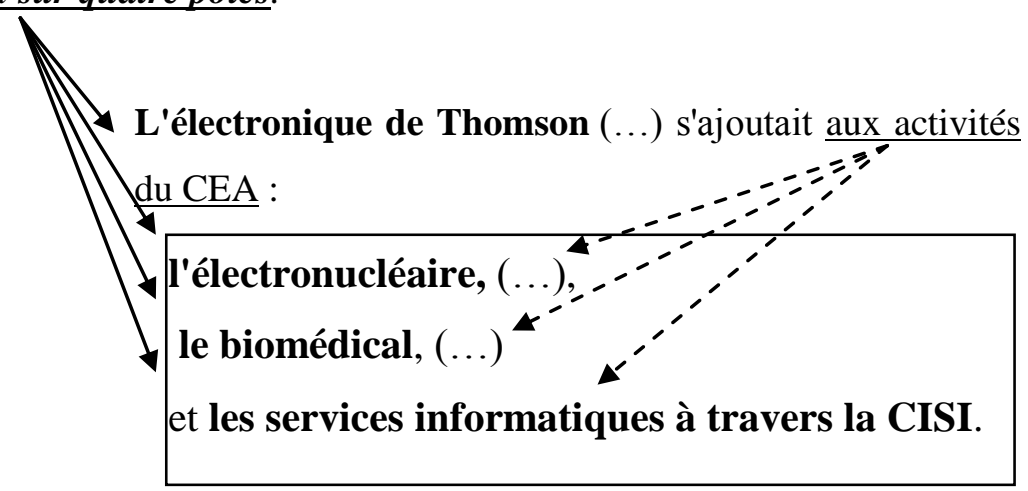

Ce schéma n'a pas résisté à l'épreuve des hommes et des faits.

A ces formes de progression peu contraintes syntaxiquement, on peut opposer la nécessité du parallélisme de structure associé au fonctionnement des cadres. Les cadres engendrent en effet des séries qui sont exprimées par des éléments périphériques en tête de phrase et dont le fonctionnement repose sur le parallélisme entre les 'slots' en position initiale.

Il faut noter encore que l'on peut facilement glisser d'un mode de structuration à l'autre. Par exemple, un critère spatial peut être introduit en position rhématique et être opposé dans la suite du texte à un second critère spatial introduit comme cadre (comme en [49]).

[49] Les vendanges ont commencé dans le midi depuis fin aout. En Alsace, on attend encore les premières gelées pour lancer les opérations.

Il faut donc retenir que nous avons affaire à deux types de structuration proches mais servant des buts communicationnels très différents : les informations véhiculées par les cadres servent à guider l'interprétation et à délimiter des blocs homogènes par rapport au critère que fixent les adverbiaux introducteurs. Les informations véhiculées dans la zone rhématique peuvent donner lieu à un développement du texte en sous-thèmes dépendants, mais cette dépendance se fait principalement par la cohésion lexicale entre les sous-thèmes élaborant un hyperrhème.

Considérons maintenant les exemples [50] et [51] cités par Crompton (2006 : 259). Ces deux phrases figurent dans les «introductory sections » de deux textes. Sans donner la suite de ces phrases, l'auteur affirme que la portée de ces adverbiaux spatiaux se prolonge apparemment jusqu'à la fin du texte.

[50] (26) You must work and be patient before you succeed in this country

'Vous devez travailler et être patients avant de réussir dans ce pays'

[51] (27) It is crunch time again in Northern Ireland

'C'est à nouveau un moment décisif en Irlande du Nord' 
Nous voudrions souligner que dans ces deux cas, les adverbiaux ne sont pas périphériques, mais selon notre classification, informationnellement requis. Ils font partie de l'information rhématique dans les deux énoncés. En [50], l'emploi du démonstratif this permet de penser que le pays a déjà été nommé, qu'il a peut-être même été introduit en position thématique. Il n'y a rien de surprenant à ce que le texte progresse au sujet de ce pays dont l'auteur précise qu'il s'agit des Etats-Unis. On peut ainsi imaginer que this country ne remplit pas un rôle de setting ou cadre dans lequel serait localisée une série d'événements mais au contraire qu'il fait entièrement partie de la trame thématique du texte qui se construit. En effet, il n'est pas introduit pour localiser un événement mais comme support de ses propres caractéristiques. En [51], l'adverbial locatif ne sert pas non plus à localiser un événement, au contraire l'Irlande du Nord est là aussi sans doute le thème central du texte (non pas en tant qu'entité géographique mais en tant qu'entité politique, en tant que lieu de conflits ancestraux etc.).

En soutenant que les adverbiaux en fin de phrase peuvent tout autant étendre leur portées sur la suite du texte que les adverbiaux en position initiale, nous nous demandons si Crompton n'amalgame pas les deux types de structuration que nous avons tenté de distinguer: structuration cadrative et progression thématique.

\section{Conclusion}

Nous avons, dans cet article, étudié les corrélations entre la position phrastique (initiale vs. finale), les valeurs sémantiques, les fonctions syntaxiques et les fonctions textuelles que peuvent prendre les SP en dans et sur, dans un corpus de Presse. En observant d'abord les corrélations entre sens et position des SP, nous avons pu mettre en évidence que deux catégories sémantiques sont plus fréquemment exprimées par ces deux prépositions: les catégories de l'espace (env. 25\%) et du notionnel (env. 70\%). Pour ces deux catégories les plus fréquentes, la distribution des deux prépositions est la même $(30 \%$ en tête/ $70 \%$ en queue), à l'exception des usages spatiaux de sur qui apparaissent autant en tête qu'en queue. Parmi les emplois notionnels, on a noté, pour dans, une prévalence des usages exprimant une 'localisation' (dans le domaine notionnel) (68\%), et, pour sur, une prévalence des usages exprimant 'l'à propos' (40\%). On a aussi observé que dans ce domaine notionnel, les deux prépositions présentent des emplois spécifiques : les valeurs 'à propos' et 'quantification' sont propres à la préposition sur, et la valeur 'support' est propre à la préposition dans. Et on a aussi vu que seules les valeurs 'support' et 'quantification' ont une nette préférence à 
apparaitre en tête $(75 \%)$. Pour toutes les autres valeurs, la proportion des occurrences en tête varie grosso-modo entre 20 et $30 \%$.

Nous avons ensuite précisé, sur la base de critères lexico-syntaxiques et sémanticopragmatiques, les statuts d'adjoints et d'arguments que peuvent prendre les SP pour observer cette fois-ci les corrélations entre sens, fonction syntaxique et position. Nous avons proposé une gradation des différents statuts d'argument et d'adjoint qui reflète différentes contraintes de dépendance vis-à-vis du prédicat, de la prédication ou de la proposition. Nous avons distingués, en les étageant du plus intégré au moins intégré : les arguments requis et régis, les arguments seulement régis, les arguments seulement requis, les arguments des constructions figées, les adjoints référentiellement requis, les adjoints informationnellement requis et les adjoints périphériques. Nous avons vu que, contrairement aux adjoints informationnellement requis ( $40 \%$ en tête), les SP proprement périphériques sont plus fréquents en tête (62\%), de même que les adjoints référentiellement requis (75\% en tête).

De plus, en faisant intervenir la composante sémantique, on a pu observer que les valeurs 'énonciatif' et 'temporel' sont intrinsèquement 'circonstantielles' - elles apparaissent entre $91 \%$ et $100 \%$ dans le statut d'adjoint, alors que les valeurs 'espace' et 'notionnel' apparaissent respectivement à $72 \%$ et $56 \%$ en statut d'adjoint. On a donc noté que, pour ces valeurs, le statut syntaxique est plus fortement tributaire de la position. La position initiale établit, d'une certaine façon, le statut circonstanciel du SP qui, en zone postverbale, est toujours susceptible de se rattacher localement à un constituant de cette zone. Les cas d'ambiguïté sont en effet légion et le positionnement en tête apparaît alors comme une stratégie efficace pour extraire un constituant de la zone d'attraction du prédicat ou de ses dépendants et lui attribuer ainsi, de façon non ambigüe, un statut d'adjoint.

Par ailleurs, dans le cas où les SP ont un statut d'adjoint, on a observé (cf. tableau 7), pour les trois types d'adjoints, une fréquence des emplois spatiaux plus élevée en queue qu'en tête de phrase, et corrélativement, une fréquence des emplois notionnels plus élevée en tête qu'en queue de phrase. Cette tendance laisse entrevoir le jeu des influences complexes entre sens, fonction et position. Si on a pu observer, d'un point de vu quantitatif, cette variation entre la position des SP adjoints et leur sens, tantôt un peu plus notionnel en tête, tantôt un peu plus spatial en queue, c'est que se dessine une affinité particulière entre la position initiale et le domaine notionnel. Il nous semble intéressant de le souligner dans la mesure où ce résultat semble aller dans le même sens que les études sur la grammaticalisation montrant l'évolution d'adverbiaux spatiaux, en position intégré, vers des marqueurs discursifs, non intégrés (cf. 
Schwenter \& Traugott. 2000, Traugott 2003, Charolles \& Pietrandrea ce volume). On a pu relever, en effet, parmi ces emplois notionnels en tête de phrase, un nombre important de syntagmes dont on pourrait dire qu'ils sont en voie de grammaticalisation, du type : Dans le cadre de ..., Dans certains cas, Dans ces conditions, Dans les faits, Dans les cas de figure, Dans le sillage de ..., Sur le fond, Sur fond de révolution féministe ..., Sur le plan des idées, Sur le terrain, Sur place, etc.

Enfin, dans la troisième partie, nous avons défendu l'hypothèse selon laquelle la position phrastique exerce une influence forte sur la capacité des SP à jouer un rôle organisationnel au niveau textuel. Nous avons distingué le rôle spécifique des SP lié à leur positionnement en tête, un rôle, en premier lieu textuel, de signalisation ou de guidage pour l'interprétation des phrases subséquentes. A ce mode de structuration, bien décrit par le terme d'indexation dans les nombreux travaux sur l'encadrement du discours initiés par (Charolles 1997), nous avons opposé un autre mode complémentaire mais tout à fait différent correspondant à un des types de progression thématique décrit par Daneš $(1970,1974)$, la progression à thème dérivé. Nous avons tenté de cerner les différences entre ces deux modes complémentaires de cohésion du discours pour apporter des arguments contre l'idée que les adverbiaux en queue ont les mêmes capacités à structurer les textes que les adverbiaux en position initiale (cf. Crompton 2006) sous prétexte qu'ils ont eux aussi une portée large sur les propositions subséquentes. Nous avons montré que dans le cas de la structuration cadrative, le SP définit l'arrière-plan qui établit un critère sémantique pour l'interprétation des phrases à venir et annule le cas échéant la portée de constituants en amont; dans les cas de progression à thème dérivé, le SP en position rhématique s'apparente à un hyper-rhème qui peut déclencher une description des sous-événements qui le composent. Les cadres fonctionnent en premier lieu au niveau textuel, les hyper-rhèmes au niveau idéationnel.

Nous avions au départ posé la question de savoir si les fonctions organisatrices dépendent (i) de la valeur propre de chaque préposition, (ii) de la valeur sémantique du syntagme prépositionnel (iii) ou de la position initiale elle-même. Au terme de cette étude, nous pouvons répondre (avec toutes les réserves liées à la limite des données que nous avons observées) que la sémantique des prépositions étudiées ne joue pas un rôle fondamental pour déterminer la capacité des adverbiaux prépositionnels à jouer un rôle textuel. Si, dans l'absolu, les prépositions statiques sont de meilleurs candidats au cadrage que les prépositions dynamiques (cf. Stosic, ce volume, Sarda \& Stosic 2007), nous n'avons pas noté de différence 
fondamentale entre les prépositions sur et dans qui ait un impact sur leur capacité à jouer un rôle discursif. En revanche, en considérant les valeurs sémantiques véhiculées par les SP, nous avons mis en évidence que les valeurs spatiales et notionnelles sont moins intrinsèquement circonstancielles que la valeur temporelle ou énonciative ${ }^{10}$ et donc plus sensibles à la position pour la détermination de leur statut syntaxique.

Enfin, en comparant des SP ayant le même statut syntaxique d'adjoint périphérique en tête et en queue, nous avons pu mettre en évidence le rôle propre de la position initiale, rôle d'index, de balise de signalisation déjà bien décrit dans la littérature. On peut parler de rôle instructionnel joué par la position initiale elle-même. De par ce rôle, placer un SP adjoint en tête résulte d'une stratégie volontaire et marquée, et de plus, le mettre en tête libère une place potentiellement rhématique en fin de phrase. En termes informationnels, le choix que l'on fait pour mettre un SP adjoint en tête ou en fin de phrase n'est pas aléatoire, il consiste à placer de l'information tantôt en background tantôt en foreground.

Mais on peut souligner qu'il n'y a pas de contradiction entre la stratégie d'organisation et de hiérarchisation des informations à travers la position initiale, et le principe avancé par Virtanen, Crucial Information First (1992: 308). Si les SP adjoints en position initiale sont mis à l'arrière plan, c'est en quelque sorte parce qu'ils fournissent une instruction première au lecteur qui peut ajuster son cadre de référence pour l'interprétation de ce qui suit. En d'autres termes, il constitue un point de départ approprié pour l'interprétation de ce qui suit. Comme le note Hasselgård (2010 : 73) le placement en position initiale d'un adjoint doit être vu comme un cas de thématisation. Elle précise, dans la tradition systémique, que ce terme est préférable à celui de fronting parce qu'il ne suggère pas que l'adjoint a subi un mouvement à partir d'une autre position dans la phrase. Il ne suggère pas non plus comme le ferait le terme 'topicalisation', que l'adjoint deviendrait le topique au sens de 'ce à propos de quoi est la phase'. Chez Halliday, le thème est le point de départ du message. Il n'est pas assimilé $a$ priori à l'information connue (comme c'est souvent le cas dans l'école de Prague (Firbas 1986 : 51). Le thème est 'speaker-oriented' : «it is where the speaker chooses to start from » (Halliday 2004: 93). Halliday ajoute cependant que la structure informationnelle non marquée étant 'given-new', on s'attend à trouver l'information connue dans le thème et l'information nouvelle dans le rhème. Cette conception du thème offre la possibilité de concevoir sans contradiction qu'un adjoint en position initiale apportent des informations nouvelles, comme c'est très souvent le cas (i.e. nouveau cadre spatial). On peut en effet

\footnotetext{
${ }^{10}$ Toutes réserves gardées compte-tenu des faibles fréquences de ces valeurs avec les deux prépositions étudiées. 
penser que c'est alors le principe CIF qui prévaut, permettant d'associer une information nouvelle à un constituant thématisé, décrit chez Halliday comme thème marqué. Pour ces raisons informationnelles et instructionnelles attachées à la position initiale, il convient de ne pas mettre sur le même pied les adjoints en position initiale et finale.

\section{Références}

CARLIER A., \& SARDA L., 2010. Le complément de la localisation spatiale : entre argument et adjoint », In Franck Neveu, Valelia Muni Toke, Thomas Klingler, Jacques Durand, Lorenz Mondada et Sophie Prévost (Eds.) CMLF 2010 - 2ème Congrès Mondial de Linguistique Française, Publié en ligne : 12 Juillet 2010, CMLF 2010, 138. http://www.linguistiquefrancaise.org/index.php?option=com_toc\&url=/articles/cml f/abs/2010/01/contents/contents.html

CARTER-THOMAS, S. 1999. Thematic networks and text type. ASp, la revue du GERAS, Vol. 23/26, 139-148.

CARTER-THOMAS, S. 2010. Texte et contexte : pour une approche fonctionnelle et empirique, Sarrebruck : Editions Universitaires Européennes

CARTER-THOMAS S. \& SARDA L. Sous presse. Identifying the semantic roles of postverbal prepositional phrases : the participant/circumstance interface. In : $L a$ transitivité en français. (dir. D. Banks et al).

CHAROLLES M., 1997. L’encadrement du discours, Document Landisco, Nancy.

CHAROLLES M., 2003. De la topicalité des adverbiaux détachés en tête de phrase, Travaux de Linguistique, 47, 11-51.

CHAROLlES M., \& S. PREVOST (eds.). 2003. Adverbiaux et topiques. Travaux de linguistique 47.

CHAROLlES M., Le DRAOULEC A., PERY-WOODLEY M-P., SARDA L. 2005. Temporal and spatial dimensions of discourse organisation, French Language Studies, 15.2: $115-130$.

CHAROLLES M., \& M-P. PERY-WOODLEY (eds.). 2005. Les adverbiaux cadratifs. Langue française 148.

COMBETTES B., 1983. Pour une grammaire textuelle. La progression thématique. De Boeck-Duculot.

CROMPTON P., 2006, "The effect of position on the discourse scope of adverbials", Text and Talk, 26(3), p. 115-130. 
DANEŠ F. 1970. One instant of Prague School Methodology: Functional Analysis of Utterance and Text, in P. Garvin (ed.), Method and Theory in Linguistics. Le Hague: Mouton: 132-146.

DANEŠ F. 1974. Functional sentence perspective and the organisation of the text, in F. Daneš (ed), Papers in Functional Sentence Perspective, Prague: Academia

DIESSEL H., 2001. The ordering distribution of main and adverbial clauses: a typological study. Language 77 (2): 433-455.

DIESSEL H., 2005. Competing motivations for the ordering of main and adverbial clauses. Linguistics 43(3): 449-470.

DIESSEL H., 2008. Iconicity of sequence: A corpus-based analysis of the positioning of temporal adverbial clauses in English. Cognitive Linguistics 19(3): 465-490.

DIK S.C., 1997. The theory of functionnal Grammar. Berlin: de Gruyter.

ENKVIST N. E., 1976. "Notes on valency, semantic scope, and thematic perspective as parameters of adverbial placement in English." In Reports on text Linguistics: Approaches to Word Order, Nils Erik Enkvist and Viljo Kohonen (eds.), 51-74. Åbo: Publications of the Research Institute of the Åbo Akademi Foundation 8.

ENKVIST N. E., 1985. A Parametric View of Word Order. In Emel Sözer (ed.), Text Connexity, Text Coherence. Hamburg: Helmut Buske, 320-336.

ENKVIST N. E., 1987. “Text strategies - single, dual, multiple.” In Language Topics: Essays in Honour of Michael Halliday, Ross Steele and Terry Threadgold (eds.), 203-211. Amsterdam/Philadelphia: Benjamins.

ENKVIST N. E., 1991. "Discourse strategies and discourse types". In Ventola,E. (ed.), Functional and systemic linguistics. Approaches and uses. New York: Mouton de Gruyter.

FIRBAS J., 1992. Functional sentence perspective in written and spoken communication. Cambridge University Press, 239 p.

FILLMORE Ch. \& KAY P., 1996. Course Book of Constructional Grammar. Manuscript, University of California at Berkeley Department of linguistics.

GIVÓN T., 1988. The pragmatics of word order: Predictability, importance and attention. In Hammond et al. (eds.) Studies in Syntactic Typology, Amsterdam: J. Benjamins, 243284.

GOLDBERG A. E. \& ACKERMAN F., 2000. The pragmatics of obligatory adjuncts. Language 77-4:798-814.

GOUTSOS D., 1996. A model of sequential relations in expository text. Text 16(4): 501-533. 
GRICE H.P., 1975. “Logic and conversation” In: Syntax and Semantics, Vol. 3, Speech Acts, ed. by Peter Cole and Jerry L. Morgan. New York: Academic Press : 41-58.

GUIMIER C., 1996. Les adverbes du français: le cas des adverbes en -ment, Paris/Gap, Ophrys, collection "L’essentiel français", $170 \mathrm{pp}$.

HAIDER, H. 2004. Pre- and postverbal adverbials in OV and VO. Lingua 114(6): 779-807. HALLIDAY M.A.K., 1994. An introduction to Functional Grammar, $2^{\text {nd }}$. Edn.., London. Arnold

HALLIDAY M.A.K., (rev. Christian M.I.M. Matthiessen) (2004): An Introduction to Functional Grammar, $3^{\text {rd }}$. edn., London, Arnold.

HASSELGÅRD H., 2010. Adjunct adverbials in English, Studies in English Language, Cambridge.

HO-DAC L-M., 2005. Deux modes de segmentation textuelle : univers de discours et chaînes de référence, Verbum, 27(3), pp 231-248.

HO-DAC L-M., 2007. La position initiale dans l'organisation du discours : une exploration en corpus, Thèse de doctorat de l'université Toulouse-le-Mirail.

HO-DAC L-M \& PÉRY-WOODLEY M-P. 2009. A data-driven study of temporal adverbials as discourse segmentation markers. In Liesbeth Degand, Cathrine Fabricius-Hansen \& Wiebke Ramm (eds). Linearisation and Segmentation in Discourse, Discours 4|2009.

HO-DAC L-M., PÉRY-WOODLEY M-P., \& TANGUY L., 2010. "Anatomie des structures énumératives", TALN 2010, Montréal, Québec, 19-23 juillet 2010. [En ligne] URL : http://www.iro.umontreal.ca/ felipe/TALN2010/Xml/Papers/all/taln2010_submission 26.pdf

MATHESIUS V., 1975. A functional analysis of present day English on a general linguistic basis, Walter de Gruyter, 228 p.

LOWE I., 1987. Sentence initial elements in English and their discourse function. Occasional Papers in Systemic Linguistics 2: 5-34.

LAMBRECHT K., 1994. Information structure and sentence form. Cambridge: Cambridge University Press.

LAZARD G. 1996. L'actance. Paris: PUF.

LEEMAN D., 2008. Prépositions du français : état des lieux. Langue Française 2008/1, $\mathrm{n}^{\circ} 157: 5-19$. 
LEEMAN D., \& VAGUER C., (eds.) 2006. La préposition en français I \& II. Modèles linguistiques (vol.53 :2006 : 1) et (vol. 54 : 2006 : II).

MELIS L., 2003. La préposition en français. Ophrys.

RAMSAY V., 1987. The functional distribution of preposed and postposed 'if' and 'when' clauses in written discourse. In Coherence and grounding in discourse, Russell S. Tomlin (ed.), 383-408. Amsterdam: John Benjamins.

SARDA L., \& CARTER-THOMAS S., 2009. Rôle informationnel et organisationnel des adverbiaux détachés en tête de phrase : étude contrastive français/anglais". Dans Florea, L., Papahagi, C., Pop. L., \& A. Curea (éds). Directions actuelles en linguistique du texte. Cluj : Casa Cartii de Stiinta. 311-328.

SARDA L. \& STOSIC D., 2007, «Les compléments spatiaux dynamiques détachés en tête : analyse des compléments en par et à travers dans la perspective de l'encadrement du discours », in FLAUX N. \& STOSIC D. (éds.), Les constructions détachées : entre langue et discours, Arras, Artois Presses Université, p. 41-56.

SCHWENTER S. \& E. TRAUGOTT. 2000. Invoking scalarity: The development of in fact, Journal of Historical Pragmatics 1, 7-25.

TALMY L., 2000. Toward a Cognitive Semantics. Vol. 1. Cambridge, MA: MIT Press.

THOMPSON S., 1985. Grammar and written discourse: Initial vs. final purpose clause in English. Text 5(1-2): 55-84.

THOMPSON S. \& LONGACRE R. E., 1985. Adverbial clauses. In: T. Shopen (ed.) Language Typology and syntactic description, vol. II, 169-205. Cambridge University Press.

TRAUGOTT E., 2003. From subjectification to intersubjectification. In R. Hickey (ed.), Motives for language change. Cambridge: Cambridge University Press, 124-139.

VAN DER SANDT R.A., (1992). Presupposition projection as anaphora resolution. Journal of Semantics, 9: 333-377.

VANPATTEN B., (Ed.) 2004. Processing Instruction: Theory, Research, and Commentary. Mahwah, New Jersey: Lawrence Erlbaum.

VIEU L., AURNAGUE M., 2007. "Part-of relations, functionality, dependence", in The categorization of spatial entities in language and cognition, M. Aurnague, M. Hickmann, L. Vieu (eds), John Benjamins (Human Cognitive Processing 20), pp. 307336. 
VERSTRAETE J-C., 2004. Initial and final position for adverbial clauses in English: the constructional basis of the discursive and syntactic differences. Linguistics 42(4): 819853.

VIRTANEN T., 1992. Discourse Functions of Adverbial Placement in English : ClauseInitial Adverbials of Time and Place in Narratives and Procedural Place Descriptions. Abo: Abo Akademi University Press.

VIRTANEN T., 2004. Point of departure: Cognitive aspects of sentence-initial adverbials. In T. Virtanen (ed.) Approaches to Cognition through Text and Discourse. (Trends in Linguistics. Studies and Monographs 147.) Berlin/New York: Mouton de Gruyter, 7997.

ZEMACH E., 1979. Four ontologies. In Pelletier F.J. Ed. Mass Terms : some philosophical problems. 63-87. Dordrecht : Reidel. 